\title{
Chapter 17 \\ Method Development for Calculating Minor Actinide Transmutation in a Fast Reactor
}

\author{
Toshikazu Takeda, Koji Fujimura, and Ryota Yamada
}

\begin{abstract}
To effectively transmute minor actinides (MAs), which have long-lived radioactivity and high decay heat, fast reactors are very promising because many minor actinides can be loaded and transmutation rates are high compared to light water reactors. With the increase of loaded minor actinides, the neutron spectrum becomes hard and core safety parameters will deteriorate. Especially, the sodium void reactivity increases with MA addition to cores. To overcome the difficulty, we propose MA transmutation fast reactors using core concepts with a sodium plenum and internal blanket region in reactor cores. Therefore, cores become complex, and calculation accuracy becomes poor. To accurately evaluate the neutronic properties such as MA transmutation rate and sodium void reactivity, we improved calculation methods. In this chapter we show new methods for calculating MA transmutation rates for each MA nuclide, for calculating the uncertainty of MA transmutation using sensitivities. A new sensitivity is derived that is defined as a relative change of core parameters relative to infinite-dilution cross sections, not effective cross sections. To eliminate bias factors in estimating core parameter uncertainties, a new method is proposed. This method is used to reduce the calculation uncertainty through the use of adjusted cross sections.
\end{abstract}

Keywords Calculation methods - Fast reactors - Minor actinide - Sensitivity - Sodium void reactivity - Transmutation

\subsection{Introduction}

The importance of nuclear energy, as a realistic option to solve the issues of the depletion of energy resources and the global environment, has been acknowledged worldwide. However, acceptance of large-scale contributions would depend on satisfaction of key drivers to enhance sustainability in terms of economics, safety,

T. Takeda $(\bowtie) \cdot R$. Yamada

Research Institute of Nuclear Engineering, University of Fukui, Fukui, Japan

e-mail: t_takeda@u-fukui.ac.jp

K. Fujimura

Hitachi Works, Hitachi-GE Nuclear Energy, Ltd., Ibaraki, Japan

(C) The Author(s) 2015

K. Nakajima (ed.), Nuclear Back-end and Transmutation Technology for Waste

Disposal, DOI 10.1007/978-4-431-55111-9_17 
adequacy of natural resources, waste reduction, nonproliferation, and public acceptance. Fast reactors with fuel recycle enhance the sustainability indices significantly, leading to the focus on sodium-cooled fast reactors (SFR) in the Generation IV International Forum (GIF) and the International Project on Innovative Nuclear Reactors and Fuel Cycles (INPRO) initiative of the International Atomic Energy Agency (IAEA).

The necessary condition for successful fast reactor deployment is the understanding and assessment of innovative technological and design options, based on both past knowledge and experience, as well as on ongoing research and technology development efforts. The severe accident at Tokyo Electric Power Company's Fukushima Dai-Ichi Nuclear Power Station caused by the Great East Japan Earthquake and tsunami on March 11, 2011 prompted all countries to redefine their fast reactor programs. To achieve the successful deployment of fast reactors, drastic safety enhancement is the most important issue to be established, especially in Japan, where the restart of nuclear power plants once these have been stopped is a serious matter of argument.

The safety aspects of fast reactors (FRs) have been reviewed [1-4] in representative countries that have developed or have a plan to develop fast reactors in the near future, especially after the Fukushima accident. These countries are improving the safety of SFRs by considering the DiD (defense in depth). The designs of SFRs should have tolerance to DBA (design basis accidents) and BDBA (beyond design basis accident) caused by internal and external events. The inherent safety and passive safety should be effectively utilized for reactor shutdown and reactor cooling. For the case of severe accidents, it is indispensable first to shut down the reactors. Furthermore, decay heat removal is also indispensable even in the case of SBO (station black out). For SFRs, natural circulation can be expected in the sodium heat transport systems and the decay heat can be removal to atmosphere by the air cooling system.

In Japan, the Ministry of Education, Culture, Sports, Science and Technology has launched a national project entitled "Technology development for the environmental burden reduction" in 2013. The present study is one of the studies adopted as the national project. The objective of the study is the efficient and safe transmutation and volume reduction of MAs with long-lived radioactivity and high decay heat contained in HLW in sodium-cooled fast reactors. We are aiming to develop MA transmutation core concepts harmonizing MA transmutation performance with core safety. The core concept is shown in Chap. 2. Also, we are aiming to improve design accuracy related to MA transmutation performance. To validate and improve design accuracy of the high safety and high MA transmutation performance of SFR cores, we developed methods for calculating transmutation rates of individual MA nuclides and estimating the uncertainty of MA transmutation.

A new definition of transmutation rates of individual MA nuclides is derived in Chap. 3. Using the definition, one can understand the physical meanings of transmutation for individual MA nuclides. Sensitivities are required to estimate the uncertainty of MA transmutation rates from cross-section errors. In Chap. 4, sensitivity calculation methods are derived. First, the sensitivity calculation method 
relative to infinite-dilution cross sections is introduced. The MA transmutation rates are burn-up properties. Thus, the sensitivity calculation method for burn-up-dependent properties is derived. Finally, we investigate how many energy groups are required in sensitivity calculations. Calculated MA transmutation rates have large uncertainties resulting from the large uncertainties in MA cross sections. To reduce these uncertainties in MA transmutation rates, we introduce a new method to reduce prediction uncertainties of MA transmutation rates in Chap. 5. In this method, we eliminate bias factors included in experiments and calculations by using ratios of the calculation to the experiment of core performance parameters. After removing the bias factors, the cross section is adjusted using measured data. The conclusions are shown in Chap. 6.

\subsection{MA Transmutation Core Concept}

MA transmutation core concepts are developed by considering the amount of MA loading and the safety-related core parameters. Increase of MA loading in the core of a SFR makes the amount of MA transmutation large, which may decrease longterm radiotoxicity and decay heat of MA. On the other hand, loading a large amount of MA into the core of a SFR increases the sodium void reactivity. Therefore, harmonization of MA transmutation and sodium void reactivity is a key issue in designing the core concepts. As an example, Fig. 17.1 shows the relationship of MA content and sodium void reactivity; when the MA content is about $10 \%$, the sodium void reactivity increases by about $1 \$$.

A homogeneous MA-loaded core of $750 \mathrm{MWe}$ was designed in the FaCT project [1, 2] (Fig. 17.2). The configuration of this core is a conventional homogeneous core and homogeneous MA loading into the core fuel increases sodium void reactivity. Therefore, MA content in the core fuel assembly is limited to less than about $5 \mathrm{wt} \%$. On the other hand, the safety issue has become more and more important since the Fukushima Daiichi NPP accident. Further, low void reactivity SFR designing has been pursued in Russia and France [5]. In this study, the coexistence of enhanced MA transmutation and zero void reactivity, that is, the harmonization of MA transmutation and core safety, is set as an objective.

Hitachi proposed an axially heterogeneous core (AHC) concept with sodium plenum $[6,7]$. It was clarified that an increase of flux level at the top of the core fuel caused by the presence of the internal blanket and decrease of the height of the inner core fuel greatly decreased sodium void reactivity. In the core concept, sodium void reactivity can be extremely reduced without disrupting core performance for normal operation. The difference in core configurations between the Hitachi AHC with sodium plenum proposed in FR '91 [6] and the ASTRID ACV [5], which has been recently studied in France, is that absorber material is loaded in the upper shield for the ASTRID ACV.

We are going to optimize the specifications of the core shown in Fig. 17.3 to realize the high MA transmutation and zero sodium void reactivity. Figure 17.4 


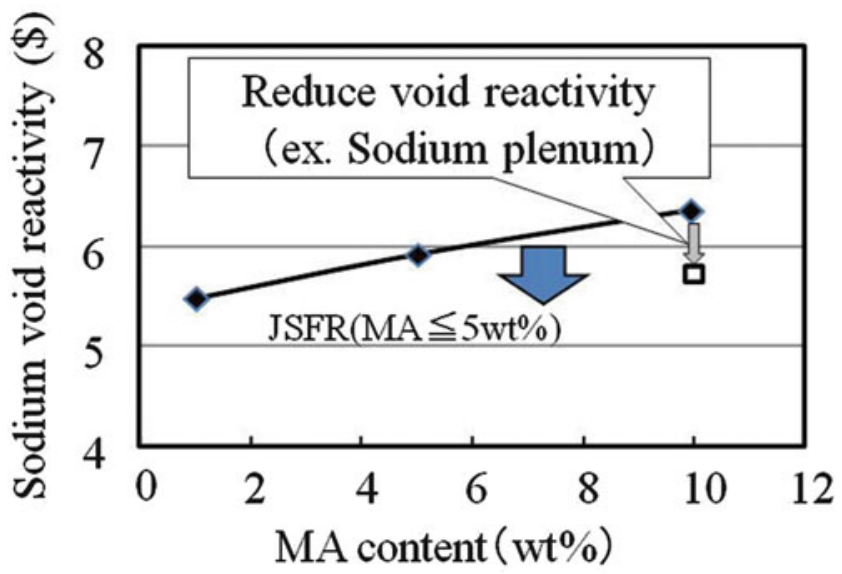

Fig. 17.1 Dependency of sodium void reactivity on minor actinide $(M A)$ content

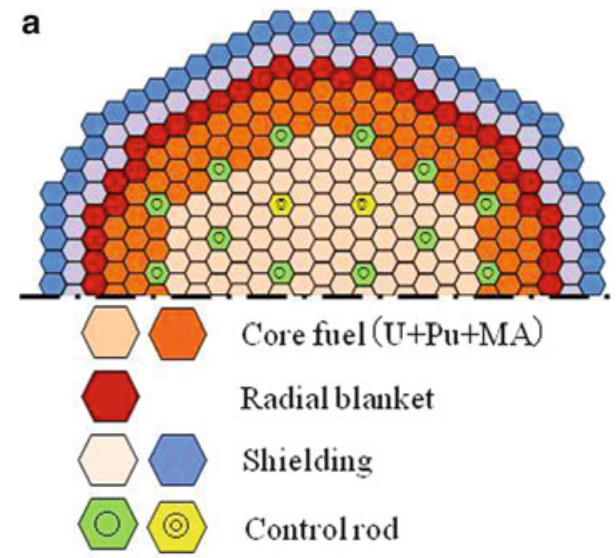

Horizontal view b

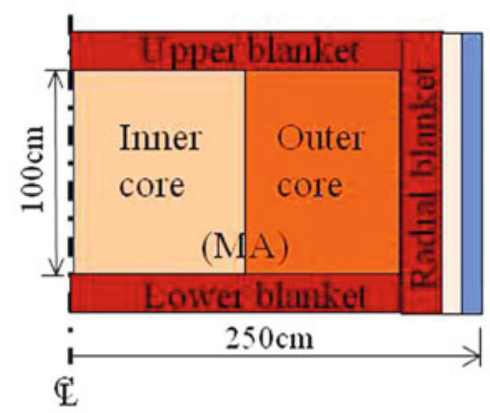

Vertical view

Fig. 17.2 Core configuration of the homogeneous MA-loaded core

shows the axial distribution of coolant density and the density coefficient of that core. The sodium density coefficient $\left(\% \Delta k / k k^{\prime} / \Delta \rho\right)$ in the sodium plenum becomes positive. On the other hand, sodium density change $(\Delta \rho)$ in the sodium plenum becomes negative because of the increase of coolant temperature for an accident such as ULOF (unprotected loss of flow accident). Therefore, net sodium void reactivity becomes negative.

The mechanism for reducing sodium void reactivity of the core is that the axial neutron leakage is largely enhanced with coolant voiding in the sodium plenum. It is known that the evaluated leakage component of sodium void reactivity with diffusion theory might be overestimated by about $50 \%$. Therefore, calculation 
Fig. 17.3 Vertical view of axially heterogeneous core with sodium plenum

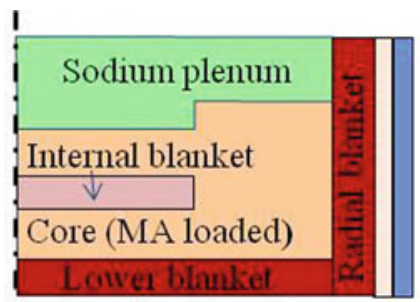

它

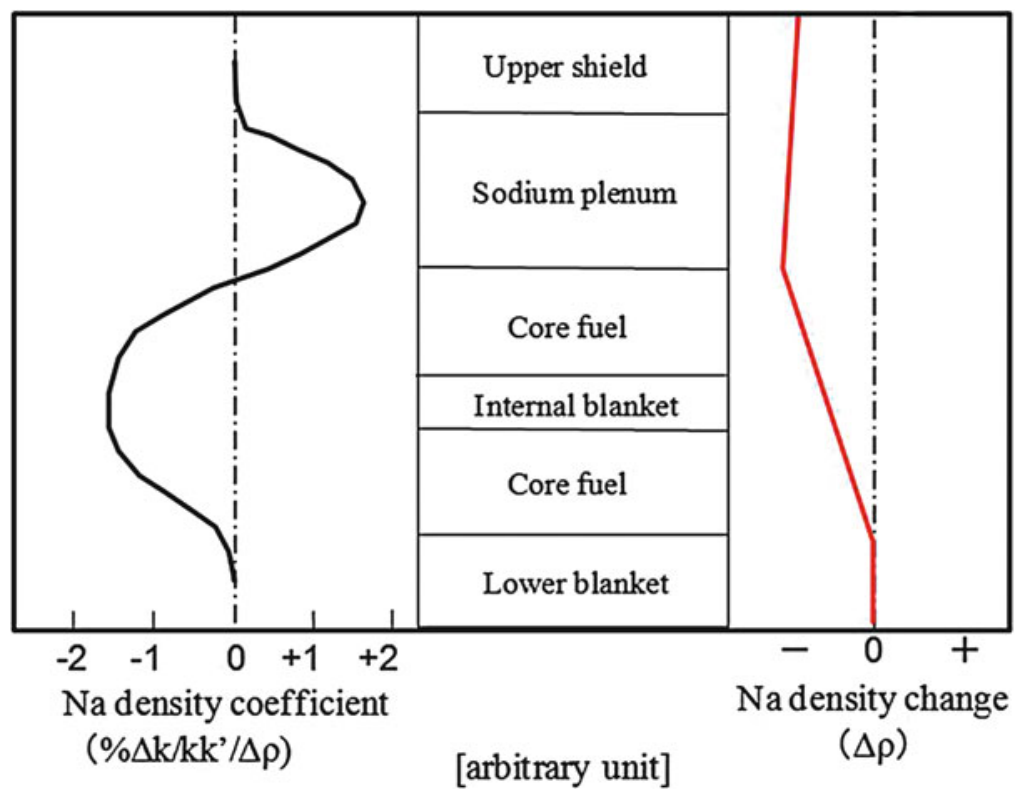

Fig. 17.4 Axial distribution of coolant density and density coefficient

accuracy for sodium void reactivity of the core with a sodium plenum might be poor. Thus, we should consider change of the neutron spectrum in the heterogeneous MA loaded core. Figure 17.5 shows the neutron spectra for MOX driver fuel without MA and $10 \%$ MA-mixed fuels in transmutation target with and without $\mathrm{Zr}-\mathrm{Hx}$. The spectrum of $10 \%$ MA-mixed fuel in transmutation target without $\mathrm{Zr}-\mathrm{Hx}$ is slightly softer than that of the MOX driver fuel without MA, because the $10 \%$ MA-mixed fuel in the transmutation target has no fissile plutonium but the MOX driver fuel includes ${ }^{239} \mathrm{Pu}$ and ${ }^{241} \mathrm{Pu}$. The neutron spectrum of the moderator mixture target fuel is clearly softer than other fuels. 


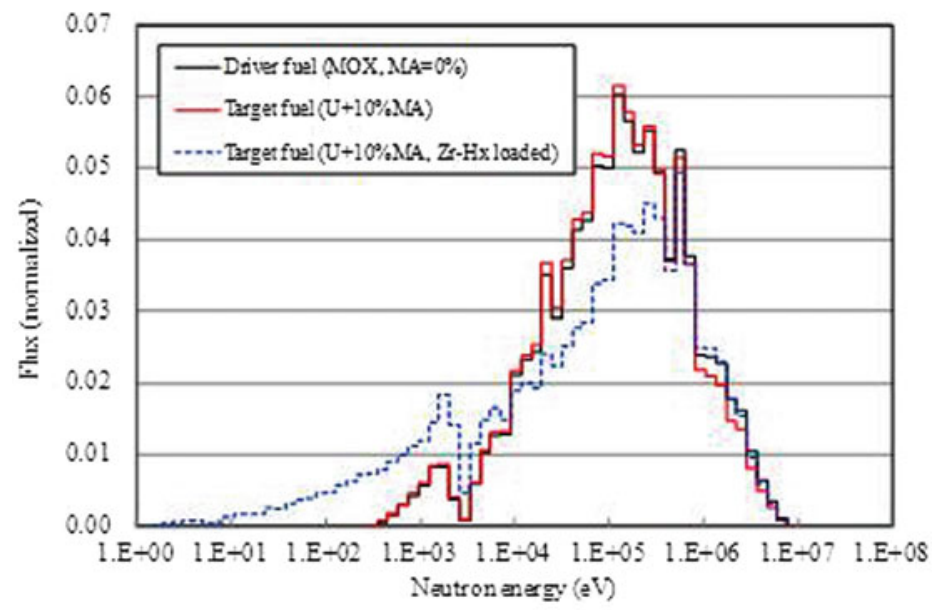

Fig. 17.5 Neutron spectra for MOX fuel and MA transmutation target fuels

\subsection{MA Transmutation Rate}

Let us first introduce a definition of transmutation rates of individual MA nuclides fueled in a reactor core. The calculation method of the transmutation rate relative to the nuclides is as follows. First, conventional burn-up calculations are carried out and burn-up-dependent flux in each region is calculated, which is used in the second step calculation. In the second step, we consider only the relevant MA in each region and perform burn-up calculations using the flux obtained in the first step.

In this second step of calculation, nuclide $k$ is produced from the original nuclide $l$. There are many passes of reactions of transmutation of the initial nuclide (shown by $N$ ) and the production of $N$ as is shown in Fig. 17.6. We can calculate the production rate of nuclide $k$ at time $T$ from the initial nuclide $l$ as

$$
P_{l k}=\widetilde{N}_{k}(T) / \widetilde{N}_{l}(0)
$$

where $\widetilde{N}_{l}(0)$ is number density of nuclide $l$ at time 0 and $\widetilde{N}_{k}(T)$ is number density of nuclide $k$ at time $T$, assuming nuclide $l$ is present alone at $t=0$. Using $\widetilde{N}_{k}(T)$, the overall fission (see Fig. 17.6) relative to the initial nuclide $l$ is calculated as

$$
\mathrm{OF}^{l}=\sum_{k} \int_{0}^{T} \sigma_{f}^{k}(t) \widetilde{N}_{k}(t) \phi(t) \mathrm{d} t
$$

where $\sigma_{f}^{k}(t)$ is fission cross section of nuclide $k$ at time $t, \phi(t)$ is neutron flux at time $t$, and $\Sigma$ is summation over all nuclides $k$ resulting from initial nuclide $l$; this 


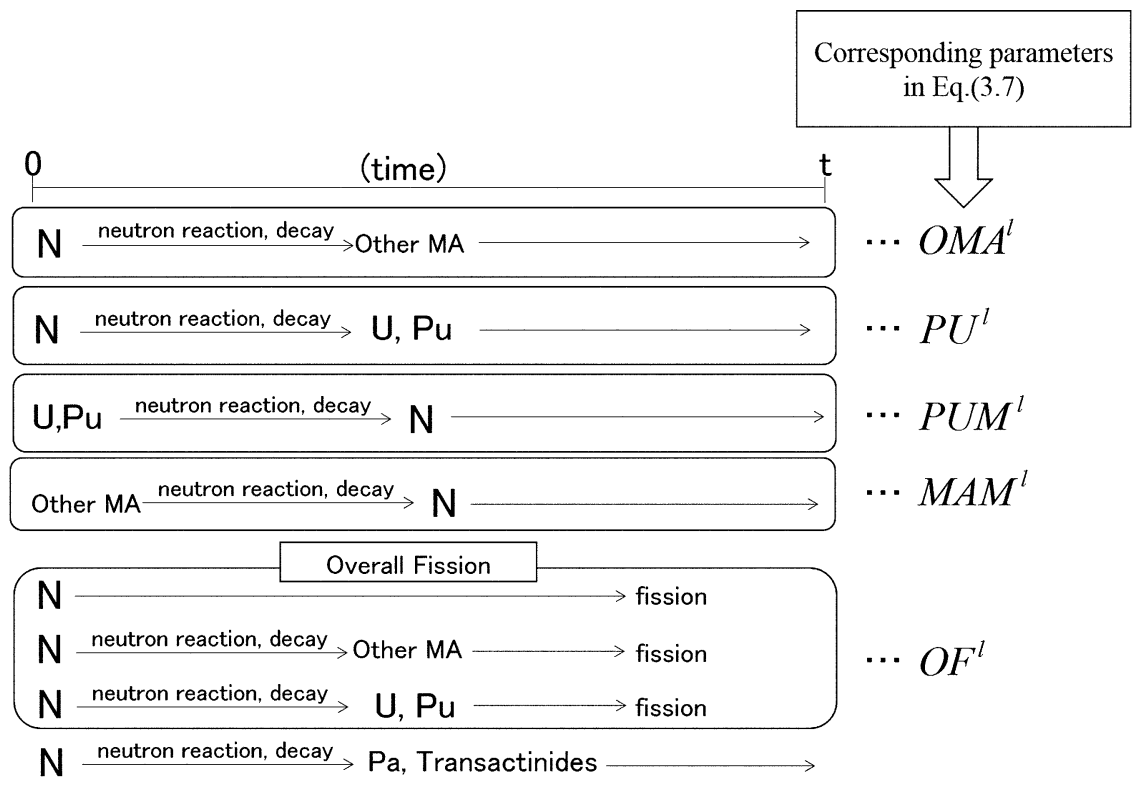

Fig. 17.6 Transmutation of initial MA nuclides and production of the MA nuclide

includes all the fissions from the initial nuclide $l$. Furthermore, the production of other MA nuclides except the initial nuclide $l$ can be calculated by

$$
\mathrm{OMA}^{l}=\widetilde{N}_{l}(0) \sum_{k \in \mathrm{MA}, k \neq l} P_{l k}
$$

The $\mathrm{Pu}$ and $\mathrm{U}$ production from nuclide $l$ is given by

$$
\mathrm{PU}^{l}=\tilde{N}_{l}(0) \sum_{k \in \mathrm{U}, \mathrm{Pu}} P_{l k}
$$

The production of MA nuclide $l$ from $\mathrm{Pu}$ and $\mathrm{U}$ is given by

$$
\mathrm{PUM}^{l}=\sum_{k \in \mathrm{U}, \mathrm{Pu}} \tilde{N}_{k}(0) P_{k l}
$$

The production of MA nuclide $l$ from other MA is given by

$$
\operatorname{MAM}^{l}=\sum_{k \in \mathrm{MA}, k \neq l} \tilde{N}_{k}(0) P_{k l}
$$

Using Eqs. (17.2), (17.3), (17.4), (17.5), and (17.6), the net transmutation of nuclide $l$ is calculated by 


$$
\mathrm{TR}^{l}=\mathrm{OF}^{l}+\mathrm{OMA}^{l}+\mathrm{PU}^{l}-\mathrm{PUM}^{l}-\mathrm{MAM}^{l}
$$

In Fig. 17.6, the individual parameters $\mathrm{OMA}^{l}, \mathrm{PU}^{l}, \mathrm{PUM}^{l}, \mathrm{MAM}^{l}$, and $\mathrm{OF}^{l}$ are shown. $\mathrm{PUM}^{l}$ and $\mathrm{MAM}^{l}$ denote the productions of the relevant MA nuclide from fuel $(\mathrm{Pu}, \mathrm{U})$ and other MA nuclides, respectively. Thus, there are minus signs in these parameters in Eq. (17.7), whereas other parameters show the elimination of the relevant MA nuclide, so the signs are positive.

When we consider the total MA transmutation for all MA nuclides, the second and the fifth terms cancel each other, so the whole transmutation is given by

$$
\mathrm{TR}=\sum_{l \in \mathrm{MA}} \mathrm{TR}^{l}=\sum_{l \in \mathrm{MA}}\left(\mathrm{OF}^{l}+\mathrm{PU}^{l}-\mathrm{PUM}^{l}\right)
$$

Therefore, we can define the MA transmutation of MA nuclide $l$ by

$$
\mathrm{TR}^{l}=\mathrm{OF}^{l}+\mathrm{PU}^{l}-\mathrm{PUM}^{l}
$$

Thus, the transmutation rate is composed of two terms: the first is the amount of incineration rate by fission and the second is the net transmutation rate to fuel $(\mathrm{U}$ and $\mathrm{Pu})$. The first fission rates of individual nuclides contain the direct fission of the relevant nuclide plus the fission of other nuclides transmuted by decays or neutron reactions as "overall fission" $\left[\mathrm{OF}^{l}\right.$ in Eq. (17.9)] (Fig. 17.7). It was found that the indirect fission contribution by ${ }^{238} \mathrm{Pu}$ and ${ }^{239} \mathrm{Pu}$ is remarkably large for nuclides ${ }^{239} \mathrm{~Np}$ and ${ }^{241} \mathrm{Am}$. The net production rates of $\mathrm{U}$ and $\mathrm{Pu}$ are calculated from the difference between the production rates of $\mathrm{U}$ and $\mathrm{Pu}$ from the relevant MA nuclide and the MA production from the initial $\mathrm{U}$ and Pu. Figure 17.7 shows the overall fission rate of ${ }^{237} \mathrm{~Np}$ in a thermal advanced pressurized water reactor (APWR) and two fast reactors, a MOX-fueled sodium-cooled fast reactor and a metal-fueled lead-cooled fast reactor [8]. In the thermal reactor, the overall fission rate is about $5 \%$ in one cycle and is very small compared with the fast reactors. In fast reactors, the direct fission of ${ }^{237} \mathrm{~Np}$ is rather large, and the ${ }^{238} \mathrm{Pu}$ fission contribution is also large. The ${ }^{239} \mathrm{Pu}$ fission contribution is small for fast reactors.

We are developing a calculation code system based on the foregoing method and are planning to apply the system to MA transmutation core design. In the core design we consider a homogeneous MA loading core, and a heterogeneous MA loading core, in which MA is loaded in special assemblies with moderators. 


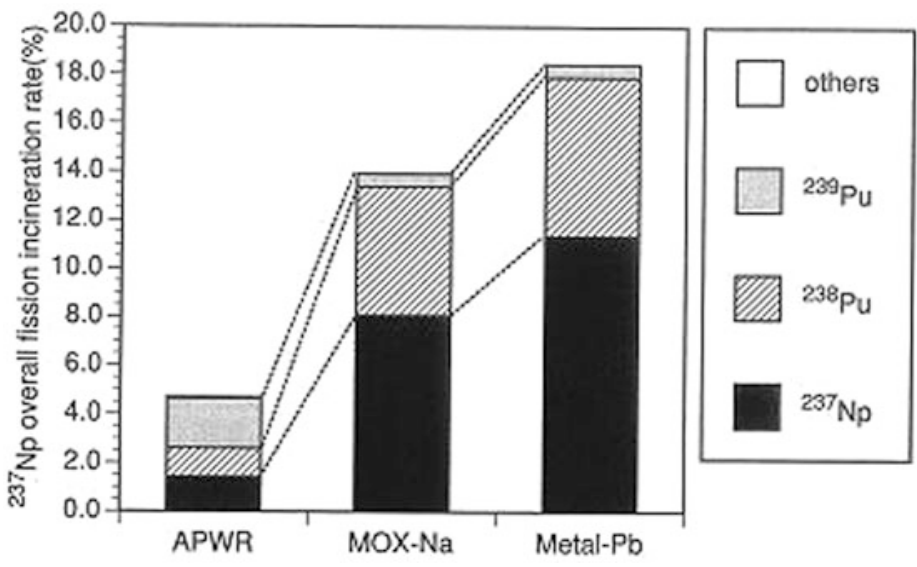

Fig. 17.7 Calculation example of overall fission rate of ${ }^{237} \mathrm{~Np}$ by developed calculation. $A P W R$ advanced pressurized water reactor

\subsection{Sensitivity Calculation Method}

\subsubsection{Sensitivity to Infinite-Dilution Cross Section}

Core characteristics such as $k_{\text {eff }}$, power distribution, and control rod worth are calculated by using effective cross section in deterministic methods. Sensitivities are usually calculated by using sensitivity calculation codes such as SAGEP [9], SAGEP-T [10], and SAINT [11]. However, the sensitivities are for relative changes of effective cross sections. Here we derive a calculation method of sensitivities relative to infinite-dilution cross sections for fast reactor analysis. Usually the effective cross sections are calculated by using the Bondarenko self-shielding factor method, the subgroup method. In that method the effective cross sections are expressed by the infinite-dilution cross section and the self-shielding factors $f$

$$
\widetilde{\sigma}=f \cdot \sigma
$$

The self-shielding factors depend on the background cross section and temperature. The background cross section for nuclide $i^{\prime}$ in a homogeneous medium is calculated by the formula

$$
\sigma_{b^{i}}=\frac{1}{N_{i^{\prime}}} \sum_{k \neq i^{\prime}} N_{k} \cdot \sigma_{t}^{k}
$$

where $N_{k}$ is the atomic number density of light nuclide $k$ and $\sigma_{t}^{k}$ is the microscopic total cross section. The sensitivity coefficient is defined by the relative changes of the core characteristics caused by the relative changes of the cross sections. Here we 
consider the following two sensitivities, the sensitivity $S$, which results from the relative change of the infinite-dilution cross sections, and the approximate sensitivity $\widetilde{S}$, which is the result of the relative change of the effective cross sections. From Eq. (17.10), the change of the effective cross section can be expressed by

$$
\frac{\mathrm{d} \widetilde{\sigma}}{\widetilde{\sigma}}=\frac{\mathrm{d} f}{f}+\frac{\mathrm{d} \sigma}{\sigma}
$$

Therefore, the improved sensitivity is expressed by using the approximate sensitivity as follows:

$$
S \equiv \frac{\mathrm{d} R / R}{\mathrm{~d} \sigma / \sigma}=\frac{\mathrm{d} R / R}{\mathrm{~d} \widetilde{\sigma} / \widetilde{\sigma}} \cdot\left(1+\frac{\mathrm{d} f / f}{\mathrm{~d} \sigma / \sigma}\right)
$$

Sensitivities and cross sections are dependent on nuclides, reaction types (such as fission, capture, and scattering), and energy groups. Here we consider the case where there is a perturbation in $\sigma$ of nuclide $i$, reaction type $j$, in energy group $g$. This perturbation causes a change in the self-shielding factor $f$ of nuclide $i^{\prime}$, reaction $j^{\prime}$, in energy group $g^{\prime}$. The second term of the right-hand side of Eq. (17.14) has to cover the contributions for all nuclides $i^{\prime}$, reaction types $j^{\prime}$, in energy groups $g^{\prime}$; therefore, we have to take the summation over $i^{\prime}, j^{\prime}$, and $g^{\prime}$. The sensitivity for the nuclide $i$, reaction type $j$, in energy group $g$ is given by

$$
S_{i, j, g}=\widetilde{S}_{i, j, g}+\sum_{i^{\prime}} \sum_{j^{\prime}} \widetilde{S}_{i^{\prime}, j^{\prime}, g^{\prime}} \cdot \frac{\mathrm{d} f_{j^{\prime}}^{i^{\prime}} / f_{j^{\prime}}^{i^{\prime}}}{\mathrm{d} \sigma_{j}^{i} / \sigma_{j}^{i}}
$$

The first term is the direct contribution to $S$; it can be calculated using the conventional tools evaluating sensitivity coefficients such as SAGEP, SAGEP-T, and SAINT. The second term represents the indirect contribution through the change of self-shielding factor. These coefficients can be calculated as follows: here we apply the resonance approximation for heavy nuclides, which is suitable for treating fast reactors with hard neutron spectra rather than light water reactors. The self-shielding effect depends on the neutron spectrum; where the neutron spectrum for the heavy nuclide $i^{\prime}$ is written as

$$
\phi(E) \propto \frac{1}{\sigma_{t}^{i^{\prime}}(E)+\sigma_{b}^{i^{\prime}}} \cdot \frac{1}{E}
$$

Equation (17.15) indicates that when $\sigma_{t}^{i^{\prime}}(E)$ and $\sigma_{b}^{i^{\prime}}$ change by the same factor, the neutron spectrum remains the same; this shows that the ratio $h$ has an effect on the neutron spectrum, and also on the self-shielding factor. Following a similar method [12], the coefficient in the second term of the right-hand side of Eq. (17.14) is called TERM and can be written as 


$$
\frac{\mathrm{d} f_{j}^{i^{\prime}}\left(h^{i^{\prime}}\right) / f_{j}^{i^{\prime}}\left(h^{i^{\prime}}\right)}{\mathrm{d} \sigma_{j}^{i}(E) / \sigma_{j}^{i}(E)}=\frac{\sigma_{j}^{i}(E)}{f_{j^{\prime}}^{i^{\prime}}\left(h^{i^{\prime}}\right)} \cdot\left\{\frac{N_{i}}{N_{i^{\prime}}}\left(1-\delta_{i i^{\prime}}\right)-\frac{\sigma_{b}^{i^{\prime}}}{\sigma_{t}^{i}(E)} \delta_{i i^{\prime}}\right\} \cdot \frac{\partial f_{j^{\prime}}^{i^{\prime}}\left(h^{i^{\prime}}\right)}{\partial \sigma_{b}^{i^{\prime}}}
$$

where the derivative of $f$ to $\sigma$ is calculated by

$$
\frac{\partial f_{j}^{i^{\prime}}\left(h^{i^{\prime}}\right)}{\partial \sigma_{b}^{i^{\prime}}}=\frac{f_{j}^{i^{\prime}}\left(\sigma_{b}^{i^{\prime}}+\Delta \sigma_{b}^{i^{\prime}}\right)-f_{j}^{i^{\prime}}\left(\sigma_{b}^{i^{\prime}}\right)}{\Delta \sigma_{b}^{i^{\prime}}}
$$

\subsubsection{Burn-up Sensitivity}

To calculate reliable MA transmutation rates, it is important to evaluate the uncertainty of calculated MA transmutation rates. The uncertainty can be calculated when the sensitivity of the MA transmutation rates to the cross sections called burnup sensitivity is known. Therefore, we developed a calculation code of burn-up sensitivity based on the generalized perturbation theory [9]. The burn-up sensitivity $\widetilde{S}$ relative to effective cross sections is calculated by

$$
\begin{aligned}
& \widetilde{S}=\frac{\mathrm{d} R / R}{\mathrm{~d} \widetilde{\sigma} / \widetilde{\sigma}} \\
& =\frac{\sigma}{R}\left[\sum_{i=1}^{I}\left\langle\int_{t_{i}}^{t_{i+1}} \mathrm{~d} t \frac{\partial R}{\partial \widetilde{\sigma}}\right\rangle+\sum_{i=1}^{I}\left\langle\int_{t_{i}}^{t_{i+1}} \mathrm{~d} t N^{*} \frac{\partial M}{\partial \widetilde{\sigma}} N\right\rangle\right. \\
& \left.+\sum_{i=1}^{I+1}\left\langle\Gamma_{i}^{*} \frac{\partial B_{i}}{\partial \widetilde{\sigma}} \phi_{i}\right\rangle+\sum_{i=1}^{I+1}\left\langle\Gamma_{i} \frac{\partial B_{i}^{*}}{\partial \widetilde{\sigma}} \phi_{i}^{*}\right\rangle-\sum_{i=1}^{I+1} P_{i}^{*}\left\langle K N \phi \frac{\partial \sigma_{f}}{\partial \widetilde{\sigma}}\right\rangle\right]
\end{aligned}
$$

In Eq. (17.18), the term containing $\frac{\partial R}{\partial \sigma}$ is called the direct term; the second term is the number density term, which represents the effect of the change of nuclide number densities caused by cross-section changes; the third term shows the effect of the change of flux from to cross-section changes; the fourth term shows the effect of the change of adjoint flux caused by cross-section changes, and the last term shows the effect of constant power production even when there are cross-section changes. The adjoint number density $N^{*}$ is calculated from the end of a burn-up period to the beginning of the period, and the generalized flux and generalized adjoint flux are calculated at each burn-up step. The adjoint number density $N^{*}$ is not continuous but has a discontinuity at each burn-up step. To calculate true burn-up sensitivities $S$ relative to infinite-dilution cross sections, we introduce $\widetilde{S}$ to Eq. (17.14) to obtain $S$. 


\subsubsection{Dependence of Sensitivities on Numbers of Energy Groups}

In sensitivity and uncertainty analysis, multi-group sensitivities are usually used, but there is no theoretical basis for the effect of number of energy groups to sensitivities. Here we derive a relationship between sensitivities calculated with different numbers of energy groups by considering the case where multi-groups are collapsed to a few groups. The sensitivity of core parameter $R$ to the microscopic cross section of nuclide $i$ and reaction $j$ in group $g$ in multi-groups is denoted by $S$ and is defined by

$$
S=\frac{\mathrm{d} R / R}{\mathrm{~d} \sigma_{i, j}^{g} / \sigma_{i, j}^{g}}
$$

The sensitivity of $R$ to microscopic cross section in few groups is given by

$$
S=\frac{\mathrm{d} R / R}{\mathrm{~d} \sigma_{i, j}^{G} / \sigma_{i, j}^{G}}
$$

Cross sections from few groups are calculated from a multi-group cross section by using neutron flux $\phi^{g}$ in group $g$ :

$$
\sigma_{i, j}^{G}=\sum_{g \in G} \sigma_{i, j}^{g} \phi^{g} / \sum_{g \in G} \phi^{g}
$$

where the summation about $g$ is performed over energy groups $g$ included in few groups $G$. Let us consider the case where multi-group cross sections change as follows:

$$
\sigma_{i, j}^{g} \rightarrow \sigma_{i, j}^{g}+\delta \sigma_{i, j}^{g}
$$

With the cross-section change, the neutron flux also changes:

$$
\phi^{g} \rightarrow \phi^{g}+\delta \phi^{g}
$$

The few group cross sections change as follows:

$$
\sigma_{i, j}^{G} \rightarrow \sigma_{i, j}^{G}+\delta \sigma_{i, j}^{G}=\sigma_{i, j}^{G}\left\{1+\frac{\sum_{g \in G}\left(\delta \phi^{g} \sigma_{i, j}^{g}+\delta \sigma_{i, j}^{g} \phi^{g}\right)}{\sum_{g \in G} \phi^{g} \sigma_{i, j}^{g}}-\frac{\sum_{g \in G} \delta \phi^{g}}{\sum_{g \in G} \phi^{g}}\right\}
$$

Therefore we obtain 


$$
\frac{\delta \sigma_{i, j}^{G}}{\sigma_{i, j}^{G}}=\frac{\sum_{g \in G}\left(\delta \phi^{g} \sigma_{i, j}^{g}+\delta \sigma_{i, j}^{g} \phi^{g}\right)}{\sum_{g \in G} \phi^{g} \sigma_{i, j}^{g}}-\frac{\sum_{g \in G} \delta \phi^{g}}{\sum_{g \in G} \phi^{g}}
$$

Here we apply the narrow resonance approximation to express the flux perturbation caused by cross-section change.

$$
\phi^{g}=\frac{C}{N_{i}\left(\sigma_{i, j}^{g}+\sigma_{0}^{g}\right)}
$$

where $C$ is a constant, $N_{i}$ is number density of nuclide $i, \sigma_{i, j}^{g}$ is microscopic total cross section of nuclide $i$, and $\sigma_{0}^{g}$ is background cross section. When using only the $j$ reaction cross section of nuclide $i, \sigma_{i, j}^{g}$, the flux perturbation is expressed by

$$
\frac{\delta \phi^{g}}{\phi^{g}}=-\frac{\delta \sigma_{i, j}^{g}}{\sigma_{i, j}^{g}+\sigma_{0}^{g}}
$$

where $\sigma_{0}^{g^{\prime}}=\sigma_{0}^{g}+\sum_{j \neq j} \sigma_{i, j}^{g}$ in the first-order approximation. Introducing the preceding equation to Eq. (17.25) leads to

$$
\begin{aligned}
& \frac{\delta \sigma_{i, j}^{G}}{\sigma_{i, j}^{G}}=\sum_{g \in G} \frac{\delta \sigma_{i, j}^{g}}{\sigma_{i, j}^{G}} \cdot \frac{\phi^{g} \sigma_{i, j}^{g}}{\sum_{g \in G} \phi^{g} \sigma_{i, j}^{g}} \\
& -\sum_{g \in G} \frac{\delta \sigma_{i, j}^{g}}{\sigma_{i, j}^{G}} \cdot \frac{\sigma_{i, j}^{g}}{\sigma_{i, j}^{g}+\sigma_{0}^{g^{\prime}}}\left\{\frac{\phi^{g} \sigma_{i, j}^{g}}{\sum_{g \in G} \phi^{g} \sigma_{i, j}^{g}}-\frac{\phi^{g}}{\sum_{g \in G} \phi^{g}}\right\}
\end{aligned}
$$

We change the multi-group cross sections $\sigma_{i, j}^{g}$ at constant rate $\alpha$ (for example, $1 \%$ ) within few groups $G$ :

$$
\frac{\delta \sigma_{i, j}^{g}}{\sigma_{i, j}^{g}}=\alpha
$$

In this case, the few-groups cross-section change is expressed by the multi-group sensitivity as follows 


$$
\frac{\delta \sigma_{i, j}^{G}}{\sigma_{i, j}^{G}}=\alpha\left(1-X^{G}\right)
$$

where $X$ is given by

$$
X^{G}=\sum_{g \in G} \frac{\sigma_{i, j}^{g}}{\sigma_{i, j}^{g}+\sigma_{0}^{g^{\prime}}}\left\{\frac{\phi^{g} \sigma_{i, j}^{g}}{\sum_{g \in G} \phi^{g} \sigma_{i, j}^{g}}-\frac{\phi^{g}}{\sum_{g \in G} \phi^{g}}\right\}
$$

Therefore, the few groups sensitivity is given by

$$
S^{G} \equiv \frac{\mathrm{d} R / R}{\mathrm{~d} \sigma_{i, j}^{G} / \sigma_{i, j}^{G}}=\sum_{g \in G} S^{g}\left(1+X^{G}\right)
$$

Thus, in general,

$$
S^{G} \neq \sum_{g \in G} S^{g}
$$

We use this relationship to choose energy groups $N(G=1-N)$ such that

$$
S^{G} \approx \sum_{g \in G} S^{g}
$$

As an example, we calculated $k_{\text {eff }}$ sensitivities in 7, 33, and 70 energy groups, and compared sensitivities. In 7 groups, the sensitivities to ${ }^{235} \mathrm{U}$ capture cross section are different from the corresponding integrated sensitivities calculated from 70 groups by $10-20 \%$ above $100 \mathrm{eV}$. However, in 33 groups, the sensitivities are different from the 70 groups result by at most $5 \%$. This result convinced us that calculations of sensitivities for 33 groups or 70 groups are sufficient.

\subsection{Reduction of Prediction Uncertainty}

To accurately calculate neutronics parameters, we have to use reliable calculation methods and nuclear data. For this purpose, we can use valuable measured data obtained from fast critical assemblies and fast reactors by applying the bias factor method [13] and the cross-section adjustment method [14]. In these two methods, it is necessary to consider that there are two kinds of errors, systematic and statistical errors, in measured and calculation errors. Here we propose a method to remove the systematic errors to improve prediction accuracy. Measured data $R_{e}$ have a systematic error $R_{\mathrm{eb}}$ and a statistical error $R_{\mathrm{es}}$ and are expressed by 


$$
R_{e}=R_{e 0}\left(1+R_{\mathrm{eb}}+R_{\mathrm{es}}\right)
$$

where $R_{e 0}$ is the true value. Also, calculated neutronics parameters $R_{c}$ are expressed by

$$
R_{c}=R_{c 0}\left(1+R_{\mathrm{cb}}+R_{\mathrm{cs}}+S \Delta \sigma\right)
$$

where $R_{c 0}$ is the true value, $R_{\mathrm{cb}}$ is systematic error, $R_{\mathrm{cs}}$ is statistical error from calculation methods, and $S \Delta \sigma$ is the error from cross-section error. To eliminate the systematic errors in measurements and calculations, we consider the ratio of measurement to calculation, called bias factors:

$$
f=\frac{R_{e}}{R_{c}}=\frac{1+R_{\mathrm{eb}}+R_{\mathrm{es}}}{1+R_{\mathrm{cb}}+R_{\mathrm{cs}}+S \Delta \sigma}
$$

Because the average of statistical errors becomes zero, the variance of $f$ becomes

$$
V(f)=V\left(R_{\mathrm{es}}\right)+V\left(R_{\mathrm{cs}}\right)+\mathrm{SWS}^{T}
$$

where $W$ is the variance of nuclear data used. In deriving Eq. (17.38) it was assumed that all the systematic and statistical errors are smaller than unity and that there is no correlation between statistical errors of measurements and calculations. From Eq. (17.38) we can say that if there is no statistical error, the bias factor $f$ is within the range of

$$
1-c \sigma<f<1+c \sigma \quad \sigma=\sqrt{V(f)}
$$

with the confidence level of $65 \%(c=1), 95 \%(c=2)$, or $99 \%(c=3)$. Therefore, if $f$ is outside the range, we can say in the foregoing confidence level, there is a systematic error of

$$
R_{\mathrm{eb}}-R_{\mathrm{cb}}=|1-f|-c \sigma
$$

For sodium void calculations, calculated values are the sum of positive nonleakage components and negative leakage components. The negative leakage components are difficult to estimate because the transport effect has to be considered in calculating the neutron steaming. Therefore, there may be a nonnegligible systematic error in the leakage term $R_{\mathrm{cb}}^{L}$ when the void pattern is leaky. By considering such a void pattern, we can discard the leakage term in systematic errors. Thus, we can determine the systematic errors. After the removal of the systematic errors, we can apply the cross-section adjustment method or the bias factor method to improve the calculation accuracy. In the cross-section adjustment method [13], the adjusted cross section is determined so as to minimize the functional $J$ 


$$
J=\left(T-T_{0}\right) W^{-1}\left(T-T_{0}\right)^{t}+\left[R_{e}-R_{c}(T)\right]\left[V_{e}+V_{m}\right]^{-1}\left[R_{e}-R_{c}(T)\right]^{t}
$$

where $W$ is the cross-section covariance data, and $V_{e}$ and $V_{m}$ are the variance of measured data and calculation method, respectively. In Eq. (17.41), we replace $R_{e}$ and $R_{c}(T)$ by

$$
\begin{aligned}
R_{e} & \rightarrow R_{e}-R_{e 0} R_{\mathrm{eb}} \\
R_{c}(T) & \rightarrow R_{c}(T)-R_{c 0} R_{\mathrm{cb}}
\end{aligned}
$$

because the true values are unknown, they are approximated by $R_{e}$ and $R_{c}$. The adjusted cross section is given by

$$
T=T_{0}+\mathrm{WG}^{t}\left[\mathrm{GWG}^{t}+V_{e}+V_{m}\right]\left[R_{e}\left(1-R_{\mathrm{eb}}\right)-R_{c}^{\prime}\left(T_{0}\right)\left(1-R_{\mathrm{cb}}\right)\right]
$$

The covariance of the adjusted cross section is expressed by

$$
W^{\prime}=W-\mathrm{WG}\left[\mathrm{GWG}^{t}+V_{e}+V_{m}\right] \mathrm{GW}
$$

This expression is the same as in [13], but we have to use the adjusted cross section shown in Eq. (17.43).

Using the adjusted cross section, the MA transmutation rate can be estimated by

$$
R_{c}^{(2)}(T)=R_{c}^{(2)}\left(T_{0}\right)+G^{(2)}\left(T-T_{0}\right)
$$

where the superscript (2) indicates the MA transmutation rate of the target reactor.

The variance, the uncertainty, of the MA transmutation rate is given by

$$
V\left[R_{c}^{(2)}(T)\right]=G^{(2)} \mathrm{W}^{\prime} \mathrm{G}^{(2) t}+V_{m}^{(2)}-\mathrm{NV}_{m}^{(12)}-V_{m}^{(12) t} N^{t}
$$

where $V_{m}^{(2)}$ is the covariance of the calculation method used for the MA transmutation rate in the target reactor core, $V_{m}^{(12)}$ is the correlation between the calculation method errors for the critical assemblies and the target core, and $N$ is defined by

$$
N=G^{(2)} \mathrm{WG}^{(1) t}\left\{G^{(1)} \mathrm{WG}^{(1) t}+V_{e}^{(1)}+V_{m}^{(1)}\right\}^{-1}
$$

We will estimate the MA transmutation amount and the uncertainties by using these methods. 


\subsection{Conclusion}

To realize the harmonization of MA transmutation and sodium void reactivity, the MA transmutation fast reactor core concept, with an internal blanket between the MA-loaded core fuel region and the sodium plenum above the core fuel, was proposed. The feature of this core concept is that sodium void reactivity can be greatly reduced without spoiling core performance for normal operation.

To accurately evaluate neutronics parameters in a MA transmutation fast reactor, we improved the calculation methods for estimating MA transmutation rates and safety-related parameters such as sodium void reactivity. For the MA transmutation rate, we introduced a definition of MA transmutation for individual MA nuclides and a method for calculating the MA transmutation rates. To evaluate the prediction accuracy of neutronics parameters, we proposed a new method that can eliminate systematic errors of measurements and calculations, and introduced a method to reduce the prediction uncertainty based on the cross-section adjustment method or the bias factor method. Furthermore, we improved the sensitivity, which is necessary to evaluate the uncertainty, by considering the effect of self-shielding.

Acknowledgments A part of the present study is the result of "Study on minor actinide transmutation using Monju data" entrusted to University of Fukui by the Ministry of Education, Culture, Sports, Science and Technology of Japan (MEXT).

Open Access This chapter is distributed under the terms of the Creative Commons Attribution Noncommercial License, which permits any noncommercial use, distribution, and reproduction in any medium, provided the original author(s) and source are credited.

\section{References}

1. Nakai R (2009) Design and assessment approach on advanced SFR safety with emphasis on the core disruptive accident issue. In: Proceedings of the international conference on fast reactor and related fuel cycles (FR09), Kyoto, 7-11 December, 2009

2. Aoto K, Uto N, Sakamoto Y, Ito T, Toda M, Kotake S (2011) Design study and R \& D progress on Japan sodium-cooled fast reactor. J Nucl Sci Technol 48:463-471

3. Beils S, Carluec B, Devictor N, Fiorini GL, Sauvage JF (2011) Safety approach and R \& D program for future French sodium-cooled fast reactors. J Nucl Sci Technol 48:510-515

4. Carluec B, Lo Point P, Mariteau P, Capelle S (2012) Severe accident countermeasure of SFR. In: Proceedings of the JAEA-IAEA international workshop on prevention and mitigation of severe accidents in sodium-cooled fast reactors, Tsuruga, 11-13 June, 2012

5. Grouiller JP et al. Transmutation in ASTRID. In: Proceedings of international conference on fast reactors and related fuel cycles: safe technologies and sustainable scenarios. FR13, Paris, March 4-7, paper IAEA-CN-199-140

6. Kawashima K et al (1991) Study of the advanced design for axially heterogeneous LMFBR cores. In: Proceedings of international conference on fast reactors and related fuel cycles, FR91, vol 1, Kyoto, October 28-November 1

7. Kawashima K et al (1992) Conceptual core design to enhance safety characteristics in MOX fueled large LMFBRs (I). Neutronics and transient safety performance characteristics. In: 
Proceedings of the international conference on design and safety of advanced nuclear power plants, ANP-92, vol 2, Tokyo, October 25-29

8. Takeda T, Narabayashi H, Hirokawa N (1998) Interpretation of transmutation rates of minor actinides in thermal and fast reactors. Ann Nucl Energy 25(9):653-665

9. Takeda T, Umano T (1985) Burnup sensitivity analysis in a fast breeder reactor. Part I: Sensitivity calculation method with generalized perturbation theory. Nucl Sci Eng 91:1-10

10. Takeda T, Asano K, Kitada T (2006) Sensitivity analysis based on transport theory. J Nucl Sci Technol 43(7):743-749

11. Nakano M, Takeda T, Takano H (1987) Sensitivity analysis of cell neutronics parameters in high-conversion light-water reactors. J Nucl Sci Technol 24(8):610-620

12. Foad B, Takeda $T$ (2014) Importance of self-shielding for improving sensitivity coefficients in light water reactors. Ann Nucl Energy 63:417-426

13. Takeda T, Yoshimura A, Kamei T, Shirakata K (1989) Prediction uncertainty evaluation method of core performance parameters in large liquid-metal fast breeder reactors. Nucl Sci Eng 103:157-165

14. Takeda T, Kamei $\mathrm{T}$ (1991) Uncertainty evaluation of burnup properties of large fast reactors using data adjustment method. Nucl Sci Eng 28(4):275-284 